\title{
Parathyroid Adenoma: A Case Report with Unusual Clinical Features
}

\author{
Urnas Islam, Jasmin Ferdous, Zeenat Jabin, Sharmin Reza and Fatima Begum \\ Natinal Institute of Nuclear Medicine and Allied Sciences (NINMAS), Dhaka, Bangladesh.
}

Address of Correspondence: Dr. Urnas Islam, Medical Officer, National Institute of Nuclear Medicine and Allied Sciences, BSMMU

Campus, Email: nitu_049@yahoo.com

\begin{abstract}
The objective of this case report is to report a rare case of parathyroid adenoma with normal parathormone and calcium levels at time of presentation. A 50 years old woman was referred to NINMAS with generalized weakness, pain in knee joints, back pain, nausea, vomiting and hypertension. High resolution neck ultrasound showed multiple, ill-defined, solid, hypoechoic nodules of varying sizes in right lobe. A small, hypoechoic, solid lesion was seen adjacent to the right lobe nodule. Serum parathormone was within normal range (39 pg/ml). Serum calcium, serum albumin, serum phosphate and ultrasound of whole abdomen reports were normal too. $99 \mathrm{mTc}$ Sestamibi scan showed tracer accumulation in early and delayed images suggesting parathyroid adenoma. Finally, right sided hemithyroidectomy was done and histopathology revealed parathyroid adenoma. Long follow up of this patient was done and serial measurement of serum calcium and PTH levels were normal throughout the period.
\end{abstract}

Bangladesh J. Nucl. Med. Vol. 22 No. 1 January 2019

Doi: https://doi.org/10.3329/bjnm.v22i1.40511

\section{INTRODUCTION}

Parathyroid adenoma is part of a spectrum of parathyroid proliferative disorder that includes parathyroid hyperplasia, parathyroid adenoma, and parathyroid carcinoma. Patients typically present with evidence of primary hyperparathyroidism, elevated serum calcium and parathyroid hormone levels. Symptoms produced by hyperplasia of the parathyroid have commonly been classified into three categories; (a) due to skeletal changes;

(b) due to urinary changes; (c) due to hypercalcemia. Most parathyroid hyperplasia results from secondary hyperparathyroidism due to renal disease. Tertiary hyperparathyroidism is the autonomous secretion of parathyroid hormone in the setting of long-standing renal disease resulting in hypercalcemia. Eighty to 85 percent of primary hyperparathyroidism is caused by parathyroid adenoma followed by primary parathyroid hyperplasia (15\%) and parathyroid carcinoma (5\%). Cystic lesions of the parathyroid gland are very uncommon, accounting for less than $0.01 \%$ of all neck masses (1). Most cystic parathyroid adenomas are located in the neck, although around $10 \%$ are found in the mediastinum (2). Functional parathyroid cysts are much more common than nonfunctional parathyroid cysts (3).

Here a rare case of parathyroid adenoma is presented with normal parathhormone (PTH) and calcium levels with Technetium-99m (99mTc) Sestamibi positive parathyroid scan.

\section{CASE REPORT}

A 50 years old woman was referred to National Institute of Nuclear Medicine and Allied Sciences (NINMAS) on 2018 for radioactive iodine uptake test, $99 \mathrm{mTc}$ thyroid scintigraphy and ultrasonography of thyroid gland. She presented with generalized weakness, pain in knee joints, back pain, nausea, vomiting and hypertension. High resolution neck ultrasound showed multiple, ill-defined, solid, hypoechoic nodules of varying sizes in right lobe. Biggest one measuring about $2.77 \mathrm{~cm} \mathrm{X} 1.67 \mathrm{~cm}$ and located in lower pole of right lobe. Another small $(0.8 \mathrm{X}$ $0.9 \mathrm{~cm}$ ) solid lesion was seen adjacent to the right lobe nodule. This one appears more hypoechoic (Figure 2). This lesion may be a parathyroid adenoma or a small thyroid nodule or enlarged lymph node. Thyroid scan report shows a photon deficient area in right lower pole, suggesting cold nodule. Serum parathormone was done twice but both reports were within normal range $(39 \mathrm{pg} / \mathrm{ml})$. Serum calcium, serum albumin, serum phosphate 
and ultrasound of whole abdomen reports were normal too. For further confirmation, parathyroid scintigraphy was done 99mTc Sestamibi and its early planer image shows focal area of increased radiotracer concentration in the region of lower pole of right lobe of thyroid gland. Rest of thyroid gland shows homogeneous radiotracer concentration and delayed image shows persistent tracer activity in the right lobe in above mentioned site with complete washout of tracer from rest of the thyroid gland, that is in the favor of parathyroid adenoma (Figure 2). Her serum calcium and PTH levels were not high and preoperative biochemical parameters were normal (serum calcium= $2.1 \mathrm{mmol} / \mathrm{L}(2.1-2.6 \mathrm{mmol} / \mathrm{L}), \mathrm{PTH}=41.8 \mathrm{pg} / \mathrm{mL}$ (11-67 pg/mL), potassium= $4.2 \mathrm{mmol} / \mathrm{L}(3.5-5.5 \mathrm{mmol} / \mathrm{L})$, sodium=141 mmol/L (135-146 mmol/L), ALT=22 U/L (up to $42 \mathrm{U} / \mathrm{L})$ and creatinine $=60 \mu \mathrm{mol} / \mathrm{L}(53-120 \mu \mathrm{mol} / \mathrm{L})$. Finally, right sided hemithyroidectomy was done and histopathology revealed parathyroid adenoma. Long follow up of this patient was done and serial measurement of serum calcium and PTH levels were normal throughout the period. Now the patient is on thyroxin and calcium supplement.
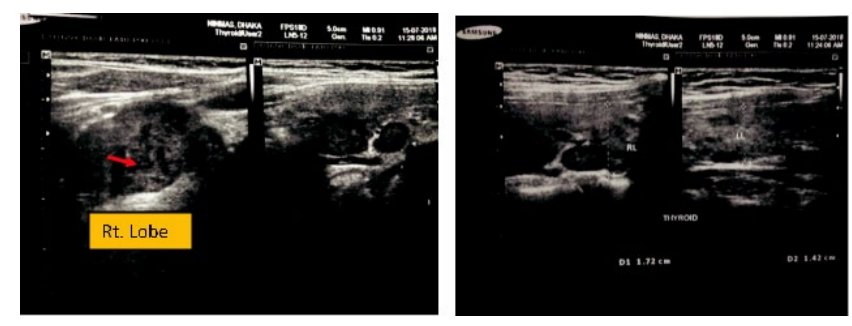

Figure 1: High resolution neck ultrasonography showing small, hypoechoic, solid lesion posterior to the right lobe nodule.
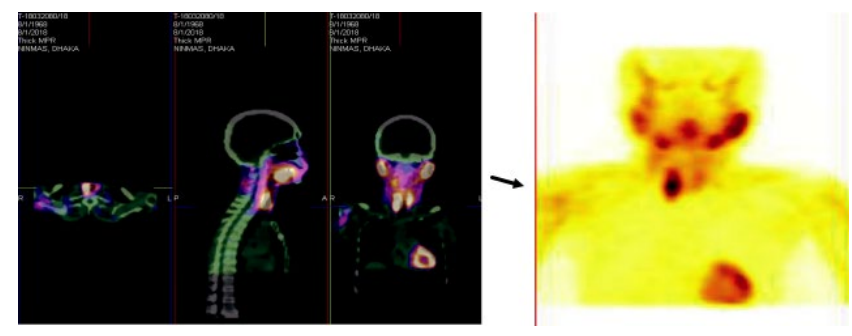

Figure 2: (a) SPECT CT images of 99mTc Sestamibi parathyroid scan showing focal uptake in right lobe area of thyroid gland in axial, sagittal and coronal sections. (b) Coronal section of $99 \mathrm{mTc}$ Sestamibi scan showing parathyroid hyperplasia

\section{DISCUSSION}

This case highlights the rare presentation of parathyroid adenoma with normal serum calcium and PTH levels with clinical features of generalized weakness, body ache, known rheumatoid arthritis and hypertension. A parathyroid adenoma is a benign tumor of parathyroid gland. It generally causes hyperparathyroidism; there are very few reports of parathyroid adenomas that were not associated with hyperparathyroidism. Parathyroid crisis is a lifethreatening emergency. It is also known in literature as acute hyperparathyroidism, parathyroid storm, para thyrotoxicosis, acute parathyroid

intoxication, parathormone intoxication, hypercalcemic crisis and calcium intoxication.

Although most cases of severe hypercalcemia are seen in malignancy (4), it is important to

emphasize that severe hypercalcemia with raised PTH is pathognomonic of primary hyperparathyroidism (5). Patients with parathyroid crisis demonstrate gastrointestinal and neurological symptoms, renal failure and cardiac rhythm abnormalities but the patient reported in this case report showed no such features as well as normal serum calcium level of $2.1 \mathrm{mmol} / \mathrm{L}$. However, gastrointestinal features like nausea and vomiting was present. She had no renal symptoms, kidney injury or cardiac rhythm abnormalities. The biochemical profile, size and texture of the solid adenoma were also rare which caused a dilemma between the differential diagnosis of parathyroid adenoma, solitary thyroid nodule or enlarged lymph node. Parathyroid scintigraphy played a vital role in the diagnosis of this patient.

Primary hyperparathyroidism is usually asymptomatic and occasionally presents with mild hypercalcemia (within $1 \mathrm{mg} / \mathrm{dL}$ above the upper limit of normal) and sometimes diagnosed incidentally on routine investigations for minor non-specific complaints. Parathyroid hyperplasia typically involves all four glands, i.e. enlargement. If one gland is significantly 
larger than the other glands it may be interpreted as parathyroid adenoma rather than hyperplasia.

Parathyroid carcinoma, on the other hand, presents with severe hypercalcemia ( $>14 \mathrm{mg} / \mathrm{dL}$ ) (6). E. Shane suggested that benign disease might have PTH level less than 2 times the upper limit of normal, whereas carcinoma may have PTH level up to 10 times the upper limit of normal (6). Robert et all suggested that PTH level less than 4 times the upper limit of normal excludes malignancy (7).

Parathyroid adenoma may rarely be cystic. The weight of parathyroid adenoma varies but in general, the mean weight for parathyroid adenoma is approximately 1 gram (the weight of a normal parathyroid gland is typically less than $50 \mathrm{mg}$ ). Cut surface is typically smooth, soft, and gray brown in color, distinguished from the yellow-brown color of normal parathyroid tissue. Microscopically, it contains subcapsular rim of compressed zone.A mixed population chief cells and oxyphil cells are arranged in sheets and supporting fibrovascular stroma. The tumour show slobulation and some cells are pleomorphic. Mitotic figures are inconspicuous $(<1 / 10 \mathrm{hpf})$ and atypical mitotic figures should not be seen in typical parathyroid adenomas.

\section{CONCLUSION}

This is a rare case of parathyroid adenoma with normal serum calcium and no rise of PTH levels. 99m Tc
Sestamibi scintigraphy serves as a valuable tool in localization of parathyroid gland. While evaluating the multinodular goiters it should always be kept in mind that parathyroid disease and parathyroid adenomas might be missed.

\section{REFERENCES}

1. Mc Kay GD, Ng TH, Morgan GJ, Chen RC: Giant functioning parathyroid cyst presenting as a retrosternal goiter. ANZJ Surg 2007, 77(4):297-304. https:// doi.org/10.1111/j.1445-2197.2007.04037.X

2. Umemori Y, Makihara S, Kotani K, Washio K. Mediastinal parathyroid cyst with tracheal constriction. Jpn J Thorac Cardiovasc Surg. 2002 Feb;50(2):85-7. PubMed PMID: 11905065.

3. Mc Coy KL, Yim JH, Zuckerbraun BS, Ogilvie JB, PeelRL,Carty SE: Cystic parathyroid lesions: functional and non-functional parathyroid cysts. Arch Surg 2009, 144:52-56. PMID: 19153325

4. Mittendorf EA, Mc Henry CR. Parathyroid carcinoma. Journal of Surgical Oncology. 2005;89:136-142. https://doi.org/10.1002/jso.20182

5. Wong P,Carmeci C, Jeffrey RB, Weigel RJ: Parathyroid crisis in a 20-year old-an unusual cause of hypercalcemic crisis. Postgrad Med J 2001,77:468-470.

6. ShaneE: Clinical review 122: parathyroid carcinoma. J Clin Endocrinol Metab 2001, 86(2):485-493. PMID: 11157996

7. Robert JH, Trombetti A, GarciaA: Primary hyperparathyroidism: can parathyroid carcinoma be anticipated on clinical and biochemical grounds? Report of nine cases and review of the literature. Ann Surg Oncol 2005,12(7):526532.https://doi.org/10.1245/ASO.2005.06.005 